EUROPEAN JOURNAL OF PURE AND APPLIED MATHEMATICS

Vol. 12, No. 3, 2019, 1337-1349

ISSN 1307-5543 - www.ejpam.com

Published by New York Business Global

\title{
Neighborhood Connected $k$-Fair Domination under some Binary Operations
}

\author{
Wardah M. Bent-Usman ${ }^{1, *}$, Rowena T. Isla ${ }^{2}$, Sergio R. Canoy, Jr. ${ }^{2}$ \\ 1 Mathematics Department, College of Natural Sciences and Mathematics, Mindanao State \\ University-Main Campus, 9700 Marawi City, Philippines \\ 2 Department of Mathematics and Statistics, College of Science and Mathematics, Cen- \\ ter for Graph Theory, Algebra, and Analysis, Premier Research Institute of Science and \\ Mathematics, Mindanao State University-Iligan Institute of Technology, 9200 Iligan City, \\ Philippines
}

\begin{abstract}
Let $G=(V(G), E(G))$ be a simple graph. A neighborhood connected $k$-fair dominating set $(n c k f d$-set) is a dominating set $S \subseteq V(G)$ such that the $|N(u) \cap S|=k$ for every $u \in V(G) \backslash S$ and the induced subgraph $\langle N(S)\rangle$ of $S$ is connected. The neighborhood connected $k$-fair domination number of $G$, denoted by $\gamma_{n c k f d}(G)$, is the minimum cardinality of an $n c k f d$-set. In this paper, we introduce and investigate the notion of neighborhood connected $k$-fair domination in graphs. We also characterize such dominating sets in the join, corona, lexicographic and Cartesian products of graphs and determine the exact values or sharp bounds of their corresponding neighborhood connected $k$-fair domination number.
\end{abstract}

2010 Mathematics Subject Classifications: 05C69, 05C76

Key Words and Phrases: $k$-fair domination, Neighborhood connected $k$-fair domination, Join, Corona, Lexicographic product, Cartesian product

\section{Introduction}

Let $G=(V(G), E(G))$ be a simple graph. A set $S \subseteq V(G)$ is a dominating set in $G$ if for every $v \in V(G) \backslash S$, there exists $u \in S$ such that $u v \in E(G)$. The minimum cardinality of a dominating set in $G$, denoted by $\gamma(G)$, is the domination number of $G$. Any dominating set in $G$ of cardinality $\gamma(G)$ is referred to as a $\gamma$-set in $G$. Arumugam and Sivagnanam [1] introduced a variation of domination called the neighborhood connected domination in graphs. A dominating set $S$ of a connected graph $G$ is called a neighborhood connected dominating set (ncd-set) if the induced subgraph $\langle N(S)\rangle$ of the open neighborhood $N(S)$ of $S$ is connected. The minimum cardinality of an $n c d$-set of $G$ is called the neighborhood

${ }^{*}$ Corresponding author.

DOI: https://doi.org/10.29020/nybg.ejpam.v12i3.3506

Email addresses: wardah_bentusman@yahoo.com (W. Bent-Usman), rowena.isla@g.msuiit.edu.ph (R. Isla), sergio.canoy@g.msuiit.edu.ph (S. Canoy) 
connected domination number of $G$ and is denoted by $\gamma_{n c}(G)$. We refer to a minimum $n c d$-set of $G$ as a $\gamma_{n c^{-}}$set.

Another domination variant is fair domination, introduced by Caro, Hansberg and Henning [3] in 2011. For an integer $k \geq 1$, a $k$-fair dominating set ( $k f d$-set) is a dominating set $S \subseteq V(G)$ such that $|N(u) \cap S|=k$ for every $u \in V(G) \backslash S$. The $k$-fair domination number of $G$, denoted by $\gamma_{k f d}(G)$, is the minimum cardinality of a $k f d$-set.

Maravilla, Isla, and Canoy [4-6] and Bent-Usman, Gomisong, and Isla [2] characterized the fair dominating, $k$-fair dominating, fair total dominating and connected $k$-fair dominating sets in the join, corona, lexicographic product, and Cartesian product of graphs and determined the bounds or exact values of the fair, $k$-fair, fair total, and connected $k$-fair domination numbers, respectively, of these graphs.

This study combines the concepts of neighborhood connected domination in graphs and of $k$-fair domination in graphs. A neighborhood connected $k$-fair dominating set (nckfd-set) is a $k$-fair dominating set $S \subseteq V(G)$ such that the induced subgraph $\langle N(S)\rangle$ is connected. The neighborhood connected k-fair domination number of $G$, denoted by $\gamma_{n c k f d}(G)$, is the minimum cardinality of an $n c k f d$-set. An $n c k f d$-set in $G$ with cardinality $\gamma_{n c k f d}(G)$ is referred to as a $\gamma_{n c k f d^{-}}$set.

\section{Preliminary Results}

Remark 1. Every nckfd-set is an ncd-set, where $k$ is a positive integer.

Remark 2. For any connected graph $G$ of order $m \geq 2$ and a positive integer $k$,

$$
1 \leq \gamma(G) \leq \gamma_{k f d}(G) \leq \gamma_{n c k f d}(G) \leq m
$$

and

$$
\gamma(G) \leq \gamma_{n c}(G) \leq \gamma_{n c k f d}(G) .
$$

The bounds given above are sharp. However, the inequalities can be attained.

To see this, consider $G=K_{4}$ and $H=C_{6}$. Clearly,

$$
1=\gamma(G)=\gamma_{1 f d}(G)=\gamma_{n c 1 f d}(G)<m .
$$

Moreover, $\gamma_{n c 4 f d}(G)=4=m$ while $\gamma(G)<\gamma_{2 f d}(G)=\gamma_{n c 2 f d}(G)=2$. Furthermore, it can be easily verified that

$$
1<2=\gamma(H)=\gamma_{1 f d}(H)<\gamma_{n c 1 f d}(H)=4 .
$$

Proposition 1. Let $G$ be a connected graph of order $n \geq 2$ and $k$ a positive integer such that $k \leq n$. Then the following hold:

(i) $\gamma_{n c k f d}(G) \geq k$. 
(ii) $\gamma_{n c k f d}(G)=k$ if and only if $G$ has an nckfd-set $S$ with $|S|=k$.

(iii) $\gamma_{n c k f d}\left(K_{n}\right)=k$.

Proof. (i) let $S$ be a $\gamma_{n c k f d}$-set. If $S=V(G)$, then $\gamma_{n c k f d}(G)=|S|=n \geq k$. Suppose $S \neq V(G)$ and let $v \in V(G) \backslash S$. Then $\left|N_{G}(v) \cap S\right|=k \leq|S|=\gamma_{n c k f d}(G)$.

(ii) Next, suppose that $\gamma_{n c k f d}(G)=k$. Then $G$ has an $n c k f d$-set $S$ with $\gamma_{n c k f d}(G)=$ $|S|=k$. For the converse, suppose that $G$ has an $n c k f d$-set $S$ with $|S|=k$. Then $\gamma_{n c k f d}(G) \leq|S|=k$. Since $\gamma_{n c k f d}(G) \geq k$, it follows that $\gamma_{n c k f d}(G)=k$. Thus, (ii) holds.

(iii) Let $G$ be $K_{n}$. Clearly, $S=V\left(K_{k}\right)$ is a $k f d$-set of $K_{n},\langle N(S)\rangle=K_{n-1}$ if $k=1$ and $\langle N(S)\rangle=K_{n}$ if $k>1$, so $S$ is an $n c k f d$-set of $K_{n}$. The result now follows by $(i i)$.

\section{Remark 3. [1]}

(i) $\gamma_{n c} \geq \gamma$

(ii) For any connected graph $G, \gamma_{n c}=1$ if and only if there exists a non-cut vertex $v$ such that $\operatorname{deg} v=n-1$. Thus, $\gamma_{n c}(G)=1$ if and only if $G=H+K_{1}$ for some connected graph $H$.

Theorem 1. [1] For any positive integer $n \geq 1, \gamma_{n c}\left(P_{n}\right)=\left\lceil\frac{n}{2}\right\rceil$.

Theorem 2. [1]

$$
\gamma_{n c}\left(C_{n}\right)= \begin{cases}\left\lceil\frac{n}{2}\right\rceil, & \text { if } n \neq 3(\bmod 4) \\ \left\lfloor\frac{n}{2}\right\rfloor, & \text { if } n \equiv 3(\bmod 4) .\end{cases}
$$

Theorem 3. For any positive integer $n \geq 1, \gamma_{n c 1 f d}\left(P_{n}\right)=\left\lceil\frac{n}{2}\right\rceil$.

Proof. Let $P_{n}=\left[v_{1}, v_{2}, \ldots, v_{n}\right]$. Clearly, the formula holds for $n=1,2,3$. Let $l$ be a positive integer. If $n=4 l$, then $S=\left\{v_{i}: i=2 a, 2 a+1, a\right.$ is odd and $\left.1 \leq a \leq 2 l-1\right\}$ is an $n c 1 f d$-set of $P_{n}$, where $\langle N(S)\rangle=P_{n}$. If $n=4 l+1$, then $S_{1}=S \cup\left\{v_{n-1}\right\}$ is an $n c 1 f d$-set of $P_{n}$, where $\left\langle N\left(S_{1}\right)\right\rangle=P_{n}$. If $n=4 l+2$, then $S_{2}=S \cup\left\{v_{n}\right\}$ is an $n c 1 f d$-set of $P_{n}$, where $\left\langle N\left(S_{2}\right)\right\rangle=P_{n-1}$. Finally, if $n=4 l+3$, then $S_{3}=S \cup\left\{v_{n-1}, v_{n}\right\}$ is an $n c 1 f d$-set of $P_{n}$, where $\left\langle N\left(S_{3}\right)\right\rangle=P_{n}$. Hence, $\gamma_{n c 1 f d}\left(P_{n}\right) \leq\left\lceil\frac{n}{2}\right\rceil$. Further, if $S$ is any $\gamma_{n c 1 f d}$-set of $P_{n}$, then $S$ is an $n c d$-set of $P_{n}$. By Remark 2 and Theorem $1,|S| \geq\left\lceil\frac{n}{2}\right\rceil$. Therefore, $\gamma_{n c 1 f d}\left(P_{n}\right)=\left\lceil\frac{n}{2}\right\rceil$.

Theorem 4. For any positive integer $n \geq 3$,

$$
\gamma_{n c 1 f d}\left(C_{n}\right)= \begin{cases}\left\lceil\frac{n}{2}\right\rceil, & \text { if } n \equiv 0 \operatorname{or} 1(\bmod 4) \\ \left\lceil\frac{n}{2}\right\rceil+1, & \text { if } n \equiv 2(\bmod 4) \\ \left\lfloor\frac{n}{2}\right\rfloor, & \text { if } n \equiv 3(\bmod 4) .\end{cases}
$$


Proof. Let $C_{n}=\left[v_{1}, v_{2}, \ldots, v_{n}, v_{1}\right]$. Clearly, $\gamma_{n c 1 f d}\left(C_{3}\right)=1$. Let $l$ be a positive integer and $n=4 l+r$, where $0 \leq r \leq 3$. Let $S=\left\{v_{i}: i=2 j, 2 j+1, j\right.$ is odd and $\left.1 \leq j \leq 2 l-1\right\}$. Let

$$
S_{1}= \begin{cases}S, & \text { if } n \equiv 0(\bmod 4) \\ S \cup\left\{v_{1}\right\}, & \text { if } n \equiv 1(\bmod 4) \\ S \cup\left\{v_{1}, v_{n}\right\}, & \text { if } n \equiv 2(\bmod 4) \\ S \cup\left\{v_{n-1}\right\}, & \text { if } n \equiv 3(\bmod 4) .\end{cases}
$$

Clearly, $S_{1}$ is a $1 f d$-set of $C_{n}$. Moreover,

$$
\left\langle N\left(S_{1}\right)\right\rangle= \begin{cases}C_{n}, & \text { if } n \supsetneqq 3(\bmod 4) \\ P_{n-1}, & \text { if } n \equiv 3(\bmod 4),\end{cases}
$$

thus, $S_{1}$ is an $n c 1 f d$-set of $C_{n}$. Hence,

$$
\gamma_{n c 1 f d}\left(C_{n}\right) \leq \begin{cases}\left\lceil\frac{n}{2}\right\rceil, & \text { if } n \equiv 0 \text { or } 1(\bmod 4) \\ \left\lceil\frac{n}{2}\right\rceil+1, & \text { if } n \equiv 2(\bmod 4) \\ \left\lfloor\frac{n}{2}\right\rfloor, & \text { if } n \equiv 3(\bmod 4) .\end{cases}
$$

Now, let $S$ be any $\gamma_{n c 1 f d}$-set of $C_{n}$. By Remark 2 and Theorem 2 ,

$$
\gamma_{n c 1 f d}\left(C_{n}\right) \geq \gamma_{n c}\left(C_{n}\right)= \begin{cases}\left\lceil\frac{n}{2}\right\rceil, & \text { if } n ¥ 3(\bmod 4) \\ \left\lfloor\frac{n}{2}\right\rfloor, & \text { if } n \equiv 3(\bmod 4) .\end{cases}
$$

If $n \equiv 0$ or $1(\bmod 4)$, then $\gamma_{n c 1 f d}(G) \geq\left\lceil\frac{n}{2}\right\rceil$. If $n \equiv 3(\bmod 4)$, then $\gamma_{n c 1 f d}(G) \geq\left\lfloor\frac{n}{2}\right\rfloor$. Moreover, when $n \equiv 2(\bmod 4)$, then $\left\langle S_{1}\right\rangle$ contains two vertices more than when $n \equiv$ $0(\bmod 4)$ and one vertex more than when $n \equiv 1(\bmod 4)$. Thus, $\gamma_{n c 1 f d}\left(C_{n}\right) \geq\left\lceil\frac{n}{2}\right\rceil+1$ if $n \equiv 2(\bmod 4)$.

The result now follows.

Theorem 5. For any nontrivial connected graph $G, \gamma_{n c 1 f d}(G)=1$ if and only if $G=$ $H+K_{1}$ for some connected graph $H$.

Proof. Suppose $\gamma_{n c 1 f d}(G)=1$. Then by Remark $2, \gamma_{n c}(G)=1$, thus $G=H+K_{1}$ for some connected graph $H$ by Remark 3. Conversely, suppose $G=H+K_{1}$ for some connected graph $H$. Let $\langle S\rangle=K_{1}=\langle\{v\}\rangle$. Then $\langle N(S)\rangle=H$ and clearly $S$ is a $\gamma_{n c 1 f d}$-set of $G$. Hence, $\gamma_{n c 1 f d}(G)=1$.

Corollary 1. Let $n$ be a positive integer. Then $\gamma_{n c 1 f d}\left(F_{n}\right)=\gamma_{n c 1 f d}\left(K_{1, n}\right)=1$ for $n \geq 1$ and $\gamma_{n c 1 f d}\left(W_{n}\right)=1$ for $n \geq 3$.

Theorem 6. Let $a$ and $b$ be positive integers such that $2 \leq a \leq b$. Then there exists $a$ connected graph $G$ such that $\gamma_{1 f d}(G)=a$ and $\gamma_{n c 1 f d}(G)=b$. 


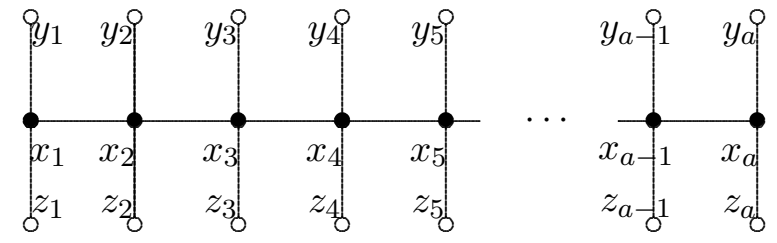

Figure 1: A graph $G$ with $\gamma_{1 f d}(G)=\gamma_{n c 1 f d}(G)=a=b$.

Proof. Consider the following cases:

Case 1. $a=b$

Let $G$ be the graph shown in Figure 1 .

It is clear that the set $A=\left\{x_{i}: i=1,2, \ldots a\right\}$ is both a $\gamma_{1 f d}$-set and a $\gamma_{n c 1 f d}$-set in $G$. It follows that $\gamma_{1 f d}(G)=\gamma_{n c 1 f d}(G)=|A|=a=b$.

Case 2. $a<b$

Subcase 1. $b=a+1$

Let $G$ be the graph shown in Figure 2. Then $A=\left\{x_{1}, x_{2}, \ldots, x_{a}\right\}$ is a $\gamma_{1 f d}$-set of $G$ and $B_{1}=A \cup\{q\}$ and $B_{2}=\left\{x_{1}, x_{2}, \ldots, x_{a-1}, w, q\right\}$ are the (only) $\gamma_{n c 1 f d^{-}}$-sets of $G$. Thus, $\gamma_{1 f d}(G)=a$ and $\gamma_{n c 1 f d}(G)=b=a+1$.

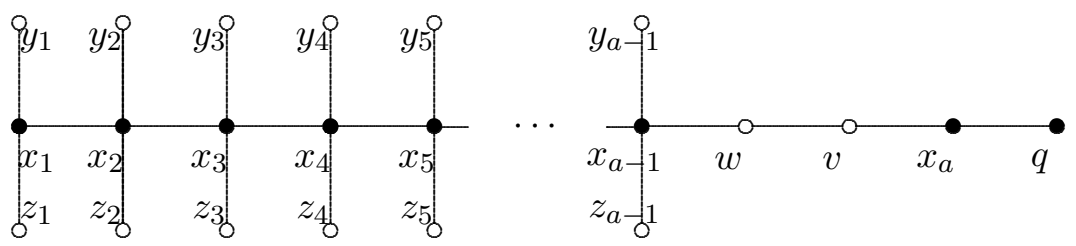

Figure 2: A graph $G$ with $\gamma_{1 f d}(G)=a$ and $\gamma_{n c 1 f d}(G)=b$ when $a<b$.

Subcase $2 . b \geq a+2$

Let $r=b-a \geq 2$. Let $H_{1}$ and $H_{2}$ be graphs such that $H_{1} \cong H_{2} \cong K_{r}$. Consider the graph $G$ in Figure 3 .

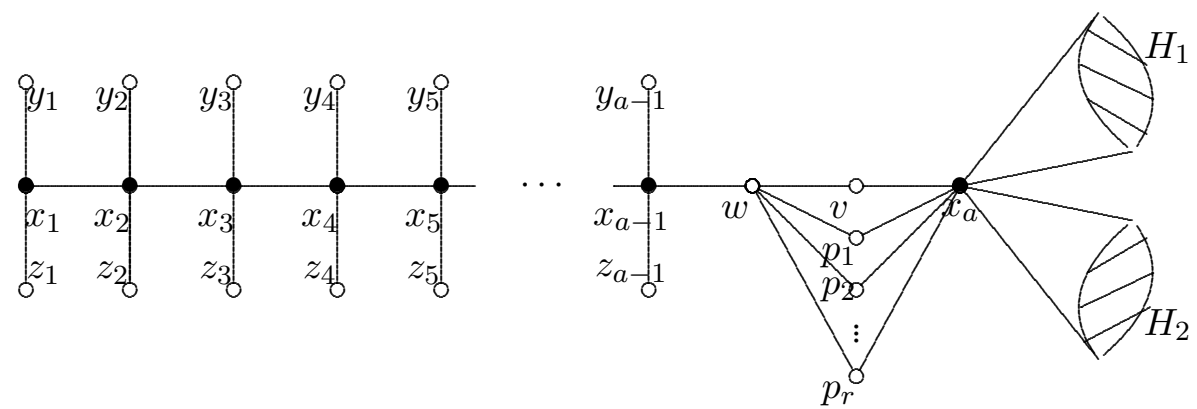

Figure 3: A graph $G$ with $\gamma_{1 f d}(G)=a$ and $\gamma_{n c 1 f d}(G)=b$ when $a<b$ and $b-a \geq 2$.

Clearly, $A_{1}=\left\{x_{1}, x_{2} \ldots, x_{a}\right\}$ is a $\gamma_{1 f d^{-}}$set of $G$. Let $B$ be a $\gamma_{n c 1 f d^{-s e t}}$ of $G$. Then 
clearly, $\left\{x_{1}, x_{2}, \ldots, x_{a-1}\right\} \subseteq B$. Suppose $x_{a} \notin B$. Then $V\left(H_{j}\right) \cap B \neq \varnothing$ for $j=1,2$, contrary to the assumption that $B$ is a $1 f d$-set. Therefore, $x_{a} \in B$. If $w \notin B$, then

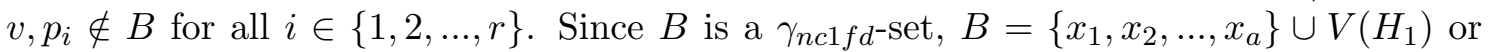
$B=\left\{x_{1}, x_{2}, \ldots, x_{a}\right\} \cup V\left(H_{2}\right)$, where $|B|=a+r=b$. Suppose that $w \in B$. Since $B$ is a $1 f d$-set, it follows that $v, p_{i} \in B$ for all $i \in\{1,2, \ldots, r\}$. Hence, $B=\left\{x_{1}, x_{2}, \ldots, x_{a}, w, v\right\} \cup$ $\left\{p_{1}, p_{2}, \ldots, p_{r}\right\}$ and $|B|=b+2$. This is not possible because $\left\{x_{1}, x_{2}, \ldots, x_{a}\right\} \cup V\left(H_{1}\right)$ is an $n c 1 f d$-set having exactly $b$ elements. Therefore, $w \notin B$ and $B=\left\{x_{1}, x_{2}, \ldots, x_{a}\right\} \cup V\left(H_{1}\right)$ or $B=\left\{x_{1}, x_{2}, \ldots, x_{a}\right\} \cup V\left(H_{2}\right)$. Accordingly, $\gamma_{1 f d}(G)=a$ and $\gamma_{n c 1 f d}(G)=b$.

Corollary 2. $\gamma_{n c 1 f d}-\gamma_{1 f d}$ can be made arbitrarily large.

Theorem 7. Let $a$ and $b$ be positive integers such that $4 \leq a \leq b$. Then there exists a connected graph $G$ such that $\gamma_{2 f d}(G)=a$ and $\gamma_{n c 2 f d}(G)=b$.

Proof. Consider the following cases:

Case 1. $a=b$

Let $G$ be the graph shown in Figure 4 . Clearly, the set $B=\left\{x_{i}: i=1,2, \ldots a\right\}$ is both a $\gamma_{2 f d}$-set and a $\gamma_{n c 2 f d}$-set in $G$. It follows that $\gamma_{2 f d}(G)=\gamma_{n c 2 f d}(G)=|B|=a=b$.

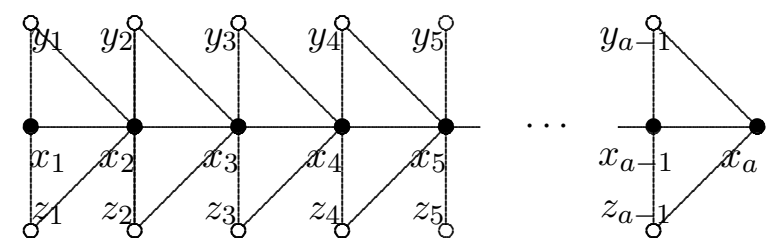

Figure 4: A graph $G$ with $\gamma_{2 f d}(G)=\gamma_{n c 2 f d}(G)=a=b$.

Case 2. $a<b$

Let $m=b-a$ and let $H_{1}, H_{2}, H_{3}$ and $H_{4}$ be graphs such that $H_{1} \cong H_{2} \cong H_{3} \cong H_{4} \cong$ $K_{m}$. Consider the graph $G$ in Figure 5 .

Clearly, $A_{1}=\left\{x_{1}, x_{2}, \ldots, x_{a}\right\}$ is a $\gamma_{2 f d}$-set of $G$. Suppose $A$ is a $\gamma_{n c 2 f d}$-set of $G$. It is easy to show that $\left\{x_{1}, x_{2}, \ldots, x_{a-2}\right\} \subseteq A$. Suppose that $x_{a-1} \notin A$. Then $y_{a-2}, z_{a-2} \in$ $A$. This implies that $y_{a-1} \notin A$ and $V\left(H_{1}\right) \cap A=\varnothing$. Hence, $\left|N_{G}\left(y_{a-1}\right) \cap A\right| \leq 1$, a contradiction. Thus, $x_{a-1} \in A$. Suppose that $x_{a} \notin A$. Then $\left|V\left(H_{3}\right) \cap A\right|=1$ and $\left|V\left(H_{4}\right) \cap A\right|=1$. It follows that $V\left(H_{1}\right) \cap A=\varnothing$ and $y_{a-1} \notin A$. This, however, implies that $N_{G}\left(y_{a-1}\right) \cap A=\left\{x_{a-1}\right\}$, a contradiction. Therefore, $A_{1} \subseteq A$. Since $\left\langle N_{G}\left(A_{1}\right)\right\rangle$ is not connected, $\left|A_{1}\right|=a<|A|$, that is, $A_{1} \neq A$. Let $v \in A \backslash A_{1}$. If $v=y_{a-1}$ or $v \in V\left(H_{1}\right)$, then $\left\{y_{a-1}\right\} \cup V\left(H_{1}\right) \subseteq A$. If $v=z_{a-1}$ or $v \in V\left(H_{2}\right)$, then $\left\{z_{a-1}\right\} \cup V\left(H_{2}\right) \subseteq A$. Let $B=A_{1} \cup\left\{y_{a-1}\right\} \cup V\left(H_{1}\right)$ or $B=A_{1} \cup\left\{z_{a-1}\right\} \cup V\left(H_{2}\right)$. Then $B$ is an $n c 2 f d$-set of $G$. Hence, $|A| \leq|B|=b+1$. Now, if $v \in V\left(H_{3}\right)$, then $V\left(H_{3}\right) \subseteq A$. Similarly, if $v \in V\left(H_{4}\right)$, then $V\left(H_{4}\right) \subseteq A$. Let $A^{*}=A_{1} \cup V\left(H_{3}\right)$ or $A^{*}=A_{1} \cup V\left(H_{4}\right)$. Then $A^{*}$ is an $n c 2 f d$-set of $G$ and $\left|A^{*}\right|=b<|B|$. Since such $v$ exists, $A=A_{1} \cup V\left(H_{3}\right)$ or $A=A_{1} \cup V\left(H_{4}\right)$. Therefore, $\gamma_{2 f d}(G)=\left|A_{1}\right|=a$ and $\gamma_{n c 2 f d}(G)=|A|=b$. 


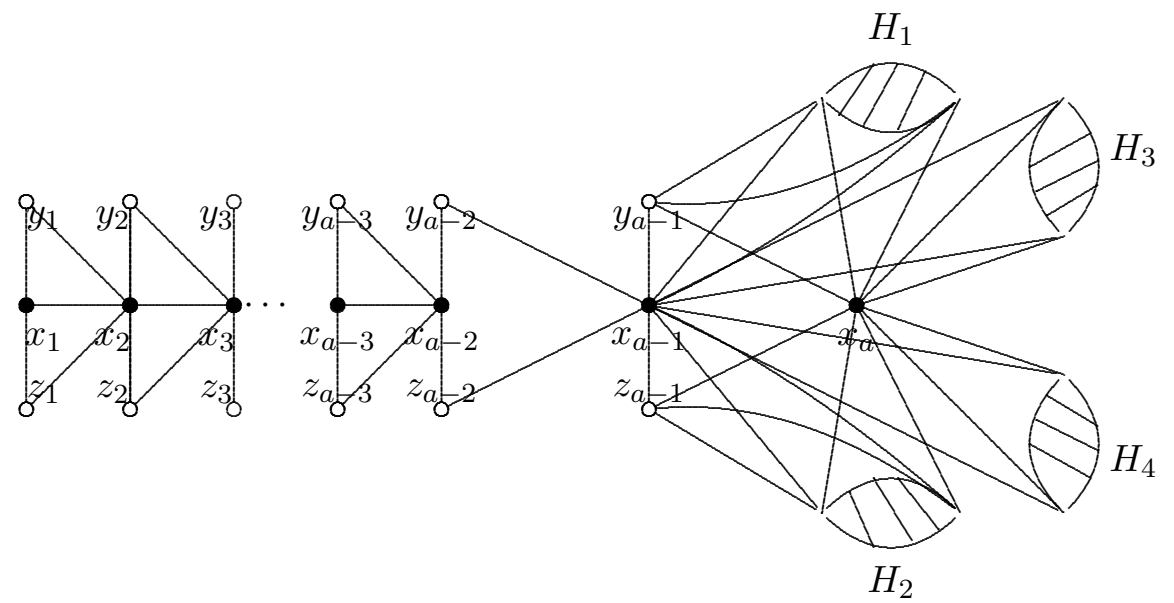

Figure 5: A graph $G$ with $\gamma_{2 f d}(G)=a$ and $\gamma_{n c 2 f d}(G)=b$ when $a<b$.

Corollary 3. $\gamma_{n c 2 f d}-\gamma_{2 f d}$ can be made arbitrarily large.

Theorem 8. [6] Let $G$ and $H$ be nontrivial connected graphs of orders $m$ and $n$, respectively, and $k$ a positive integer with $1 \leq k \leq \max \{m, n\}$. Then $S \subseteq V(G+H)$ is a $k f d$-set of $G+H$ if and only if one of the following holds:

(a) $S=V(G+H)$.

(b) $S \subseteq V(G),|S|=k$ and $S$ is a $k f d$-set in $G$.

(c) $S \subseteq V(H),|S|=k$ and $S$ is a $k f d$-set in $H$.

(d) $S=S_{G} \cup S_{H}$, where $S_{G}$ is a $\left(k-\left|S_{H}\right|\right) f d$-set of $G$ and $S_{H}$ is a $\left(k-\left|S_{G}\right|\right) f d$-set in $H$.

(e) $S=V(G) \cup T$, where $|V(G)|=m<k$ and $T$ is a $(k-m) f d$-set in $H$.

(f) $S=D \cup V(H)$, where $|V(H)|=n<k$ and $D$ is a $(k-n) f d$-set in $G$.

Theorem 9. [6] Let $G$ and $H$ be nontrivial connected graphs and let $k$ be a positive integer with $k \leq|V(H)|$. Then $C \subseteq V(G \circ H)$ is a $k f d$-set in $G \circ H$ if and only if one of the following holds:

(a) $C=V(G) \cup B$, where $B=\varnothing(k=1)$ or $B=\bigcup_{v \in V(G)} S_{v}$, where each $S_{v}$ is a $(k-1) f d$-set of $H^{v}(k \geq 2)$.

(b) $C=\bigcup_{v \in V(G)} S_{v}$, where each $S_{v}$ is a kfd-set of $H^{v}$ and $\left|S_{v}\right|=k$.

Theorem 10. [6] Let $G$ and $H$ be nontrivial connected graphs. Then $C=\bigcup_{x \in S}\left(\{x\} \times T_{x}\right) \subseteq$ $V(G[H])$ is a kfd-set in $G[H]$ if and only if the following hold: 
(i) $S$ is a dominating set in $G$.

(ii) For each $x \in S \cap N_{G}(S), T_{x}=V(H)$ and $|V(H)|=r \leq k$ whenever $C \neq V(G[H])$ or $T_{x}$ is an $r f d$-set and $\sum_{z \in N_{G}(x) \cap S}\left|T_{z}\right|=k-r$.

(iii) For each $x \in S \backslash N_{G}(S), T_{x}=V(H)$ and $|V(H)| \leq k$ or $\left|T_{x}\right|=k$ and $T_{x}$ is a $k f d$-set in $H$.

(iv) For each $y \in V(G) \backslash S, \sum_{v \in N_{G}(y) \cap S}\left|T_{v}\right|=k$.

Corollary 4. [6] Let $G$ and $H$ be nontrivial connected graphs of orders $m$ and $n$, respectively, and $k$ a positive integer with $1 \leq k \leq \min \{m, n\}$. If $D$ is a $k f d$-set in $H$, then $V(G) \times D$ is a $k f d$-set in $G \square H$.

\section{Neighborhood Connected $k$-Fair Domination in the Join of Graphs}

The join $G+H$ of two graphs $G$ and $H$ is the graph with vertex set $V(G+H)=$ $V(G) \cup V(H)$ and edge set $E(G+H)=E(G) \cup E(H) \cup\{u v: u \in V(G), v \in V(H)\}$.

Theorem 11. Let $G$ and $H$ be nontrivial connected graphs of orders $m$ and $n$, respectively, and $k$ a positive integer with $1 \leq k \leq \max \{m, n\}$. Then $S \subseteq V(G+H)$ is an nckfd-set in $G+H$ if and only if $S$ is a kfd-set in $G+H$.

Proof. Let $S \subseteq V(G+H)$ be an $n c k f d$-set in $G+H$. Then by definition, $S$ is a $k f d$-set in $G+H$.

For the converse, suppose $S \subseteq V(G+H)$ is a $k f d$-set in $G+H$. Then at least one of Statements $(a)$ to $(f)$ of Theorem 8 holds. We claim that $\langle N(S)\rangle$ is connected. If Statement $(a)$ holds, that is, $S=V(G+H)$, then we are done. Suppose Statement $(b)$ holds; that is, $S \subseteq V(G),|S|=k$ and $S$ is a $k f d$-set of $G$. Then $\left\langle N_{G+H}(S)\right\rangle=\left\langle N_{G}(S)\right\rangle+H$, which is connected. Similarly, if Statement (c) holds, then $\left\langle N_{G+H}(S)\right\rangle=G+\left\langle N_{H}(S)\right\rangle$ is connected. Suppose Statement $(d)$ holds; that is, $S=S_{G} \cup S_{H}$, where $S_{G}$ is a $\left(k-\left|S_{H}\right|\right) f d$ set in $G$ and $S_{H}$ is a $\left(k-\left|S_{G}\right|\right) f d$-set in $H$. Then $N_{G+H}(S)=N_{G+H}\left(S_{G}\right) \cup N_{G+H}\left(S_{H}\right)=$ $\left[N_{G}\left(S_{G}\right) \cup V(H)\right] \cup\left[N_{H}\left(S_{H}\right) \cup V(G)\right]=V(G+H)$. Hence, $\left\langle N_{G+H}(S)\right\rangle=G+H$ is connected. Suppose Statement (e) holds; that is, $S=V(G) \cup T$, where $|V(G)|=m<k$ and $T$ is a $(k-m) f d$-set in $H$. Then $N_{G+H}(S)=N_{G+H}(V(G)) \cup N_{G+H}(T)=V(H) \cup[V(G) \cup$ $\left.N_{H}(T)\right]=V(G) \cup V(H)$. Thus, $\left\langle N_{G+H}(S)\right\rangle=G+H$ is connected. Similarly, if Statement $(f)$ holds, that is, $S=D \cup V(H)$, where $|V(H)|=n<k$ and $D$ is a $(k-n) f d$-set in $G$, then $\left\langle N_{G+H}(S)\right\rangle=G+H$ is connected. Therefore, $S$ is an $n c k f d$-set in $G+H$.

The next result immediately follows from Theorem 11.

Corollary 5. Let $G$ and $H$ be nontrivial connected graphs of orders $m$ and $n$, respectively, and $k$ a positive integer with $1 \leq k \leq \max \{m, n\}$. Then, $\gamma_{n c k f d}(G+H)=\gamma_{k f d}(G+H)$. In particular, if $G$ or $H$ has a $k f d$-set $S$ with $|S|=k$, then $\gamma_{n c k f d}(G+H)=k$. 


\section{Neighborhood Connected $k$-Fair Domination in the Corona of Graphs}

The corona of two graphs $G$ and $H$, denoted by $G \circ H$, is the graph obtained by taking one copy of $G$ of order $n$ and $n$ copies of $H$, and then joining the $i$-th vertex of $G$ to every vertex in the $i$-th copy of $H$. For every $v \in V(G)$, we denote by $H^{v}$ the copy of $H$ whose vertices are joined or attached to the vertex $v$. For each $v \in V(G)$, the subgraph $\langle v\rangle+H^{v}$ of $G \circ H$ will be denoted by $v+H^{v}$.

Theorem 12. Let $G$ and $H$ be nontrivial connected graphs, and let $k$ be a positive integer with $2 \leq k \leq|V(H)|$. Then $C \subseteq V(G \circ H)$ is an nckfd-set in $G \circ H$ if and only if $C$ is a $k f d$-set in $G \circ H$.

Proof. If $C$ is an $n c k f d$-set in $G \circ H$, then $C$ is a $k f d$-set in $G \circ H$.

Conversely, let $C$ be a $k f d$-set in $G \circ H$. Then by Theorem 9 ,

(a) $C=V(G) \cup B$, where $B=\varnothing$ when $k=1$ and $B=\bigcup_{v \in V(G)} S_{v}$, where each $S_{v}$ is a $(k-1) f d$-set of $H^{v}$ when $k \geq 2$, or

(b) $C=\bigcup_{v \in V(G)} S_{v}$, where each $S_{v}$ is a $k f d$-set of $H^{v}$ and $\left|S_{v}\right|=k$.

We claim that $\langle N(C)\rangle$ is connected. Suppose Condition $(a)$ holds. Suppose further that $B=\varnothing$. Then $C$ is a $1 f d$-set and $\langle N(C)\rangle=G \circ H$, which is connected. We next assume that $B=\bigcup_{v \in V(G)} S_{v}$, where each $S_{v}$ is a $(k-1) f d$-set in $H^{v}$. Then $\langle N(C)\rangle=\left\langle\bigcup_{v \in V(G)}\left(v+H^{v}\right)\right\rangle=$ $G \circ H$ is connected. Suppose Condition $(b)$ holds. Then $\langle N(C)\rangle=\left\langle\bigcup_{v \in V(G)}\left(\{v\} \cup N_{H^{v}}\left(S_{v}\right)\right)\right\rangle$ which is connected. Therefore, $C$ is an $n c k f d$-set in $G \circ H$.

Corollary 6. Let $G$ and $H$ be nontrivial connected graphs of orders $m$ and $n$, respectively, and let $k$ be a positive integer with $1 \leq k \leq n$. Then $\gamma_{n c 1 f d}(G \circ H)=m$. For $k \geq 2$,

$$
\gamma_{n c k f d}(G \circ H)= \begin{cases}m k, & \text { if } H \text { has a kfd-set with }|S|=k \\ m\left(1+\gamma_{(k-1) f d}(H),\right. & \text { if } H \text { has no kfd-set with }|S|=k .\end{cases}
$$

Proof. This immediately follows from Theorem 12 (and its proof) .

\section{Neighborhood Connected $k$-Fair Domination in the Lexicographic Product of Graphs}

The lexicographic product of two graphs $G$ and $H$, denoted by $G[H]$, is the graph with vertex set $V(G[H])=V(G) \times V(H)$ and edge set $E(G[H])$ satisfying the following conditions: $\left(u_{1}, v_{1}\right)\left(u_{2}, v_{2}\right) \in E(G[H])$ if and only if either $u_{1} u_{2} \in E(G)$ or $u_{1}=u_{2}$ and $v_{1} v_{2} \in E(H)$. 
Theorem 13. Let $G$ and $H$ be nontrivial connected graphs of orders $m$ and $n$, respectively, and $k$ a positive integer with $1 \leq k \leq \max \{m, n\}$. Then $C=\bigcup_{x \in S}\left(\{x\} \times T_{x}\right) \subseteq V(G[H])$ is an nckfd-set in $G[H]$ if and only if the following conditions hold:

(i) $S$ is a dominating set in $G$.

(ii) For each $x \in S \cap N_{G}(S)$ with $T_{x} \neq V(H), T_{x}$ is an $r f d$-set and $\sum_{z \in N_{G}(x) \cap S}\left|T_{z}\right|=k-r$ for some $r<k$.

(iii) For each $x \in S \backslash N_{G}(S)$ with $T_{x} \neq V(H),\left|T_{x}\right|=k$ and $T_{x}$ is a $k f d$-set in $H$.

(iv) For each $y \in V(G) \backslash S, \sum_{v \in N_{G}(y) \cap S}\left|T_{v}\right|=k$.

Proof. Suppose $C=\bigcup_{x \in S}\left(\{x\} \times T_{x}\right)$ is an $n c k f d$-set in $G[H]$. Then $C$ is a $k f d$-set in $G[H]$, hence Statements $(i)$ to $(i v)$ hold by Theorem 10 .

For the converse, suppose Statements $(i)$ to $(i v)$ hold. We claim that $\left\langle N_{G[H]}(C)\right\rangle$ is connected. Suppose $C \neq V(G[H])$. Let $(x, a),(y, b) \in N_{G[H]}(C)$ such that $(x, a) \neq(y, b)$ and $(x, a)(y, b) \notin E\left(\left\langle N_{G[H]}(C)\right\rangle\right)$. Consider the following cases:

Case 1. $x=y$

Let $z \in V(G) \cap N_{G}(x)$. If $z \in S$, pick any $c \in T_{z}$ and let $d \in N_{H}(c)$. Then $(z, d) \in N_{G[H]}(z, c) \subseteq N_{G[H]}(C)$ and $[(x, a),(z, d),(y, b)]$ is an $(x, a)-(y, b)$ geodesic in $N_{G[H]}(C)$. If $z \notin S$, then $\{z\} \times T_{z} \subseteq N_{G[H]}(C)$ since $S$ is a dominating set of $G$. Thus, $[(x, a),(z, d),(y, b)]$ is an $(x, a)-(y, b)$ geodesic in $N_{G[H]}(C)$ for all $d \in T_{z}$.

Case 2. $x \neq y$

Let $\left[x_{1}, x_{2}, \ldots, x_{k}\right]$, where $x_{1}=x$ and $x_{k}=y$, be an $x-y$ geodesic. Since $S$ is a dominating set of $G,\left(\left\{x_{j}\right\} \times T_{x_{j}}\right) \cap N_{G[H]}(C) \neq \varnothing$ for each $j \in\{2,3, \ldots, k-1\}$. Pick $\left(x_{j}, a_{j}\right) \in$ $N_{G[H]}(C)$ for each $j \in\{2,3, \ldots, k-1\}$. Then $\left[\left(x_{1}, a_{1}\right),\left(x_{2}, a_{2}\right), \ldots,\left(x_{k-1}, a_{k-1}\right),\left(x_{k}, a_{k}\right)\right]$, where $a_{1}=a$ and $a_{k}=b$, is an $(x, a)-(y, b)$ path in $N_{G[H]}(C)$.

Therefore, $\left\langle N_{G[H]}(C)\right\rangle$ is connected. Hence, $C$ is an $n c k f d$-set in $G[H]$.

The next result immediately follows from Theorem 13 and Theorem 10.

Corollary 7. Let $G$ and $H$ be nontrivial connected graphs of orders $m$ and $n$, respectively, and $k$ a positive integer with $1 \leq k \leq \max \{m, n\}$. Then $C=\bigcup_{x \in S}\left(\{x\} \times T_{x}\right) \subseteq$ $V(G[H])$ is an nckfd-set in $G[H]$ if and only if $C$ is a $k f d$-set in $G[H]$. In particular, $\gamma_{n c k f d}(G[H])=\gamma_{k f d}(G[H])$.

\section{Neighborhood Connected $k$-Fair Domination in the Cartesian Product of Graphs}

The Cartesian product of two graphs $G$ and $H$, denoted by $G \square H$, is the graph with vertex-set $V(G \square H)=V(G) \times V(H)$ and edge-set $E(G \square H)$ satisfying the following conditions: $\left(u_{1}, v_{1}\right)\left(u_{2}, v_{2}\right) \in E(G \square H)$ if and only if either $u_{1} u_{2} \in E(G)$ and $v_{1}=v_{2}$ or $u_{1}=u_{2}$ and $v_{1} v_{2} \in E(H)$. 
Theorem 14. Let $G$ and $H$ be nontrivial connected graphs. If $S_{1}$ and $S_{2}$ are $k f d$-sets in $G$ and $H$, respectively, then $C_{1}=S_{1} \times V(H)$ and $C_{2}=V(G) \times S_{2}$ are nckfd-sets in $G \square H$.

Proof. Let $S_{1}$ be a $k f d$-set in $G$. By Corollary $4, C_{1}=S_{1} \times V(H)$ is a $k f d$-set of $G \square H$. Next, let $(x, a)$ and $(y, b)$ be distinct non-adjacent vertices of $\left\langle N_{G}\left(C_{1}\right)\right\rangle$. Consider the following cases:

Case 1. $x=y$

Let $\left[a_{1}, a_{2}, \ldots, a_{k}\right]$, where $a_{1}=a$ and $a_{k}=b$, be an $a$-b geodesic. If $x \in S_{1}$, then $\{x\} \times V(H) \subseteq N_{G \square H}\left(C_{1}\right)$. It follows that $\left[\left(x, a_{1}\right),\left(x, a_{2}\right) \ldots,\left(x, a_{k}\right)\right]$ is an $(x, a)-(y, b)$ path in $\left\langle N_{G \square H}\left(C_{1}\right)\right\rangle$. If $x \notin S_{1}$, then there exists $z \in S_{1} \cap N_{G}(x)$ since $S_{1}$ is a dominating set of $G$. Since $\{z\} \times V(H) \subseteq C_{1},\{x\} \times V(H) \subseteq N_{G \square H}\left(C_{1}\right)$. Hence, $\left[\left(x, a_{1}\right),\left(x, a_{2}\right) \ldots,\left(x, a_{k}\right)\right]$ is an $(x, a)-(y, b)$ path in $\left\langle N_{G \square H}\left(C_{1}\right)\right\rangle$.

Case 2. $x \neq y$

Let $\left[x_{1}, x_{2}, \ldots, x_{k}\right]$, where $x_{1}=x$ and $x_{k}=y$, be an $x-y$ geodesic. Let $j \in\{1,2, \ldots, k\}$. If $x_{j} \in S_{1}$, then $\left\{x_{j}\right\} \times V(H) \subseteq C_{1}$; hence, $\left\{x_{j}\right\} \times V(H) \subseteq N_{G \square H}\left(C_{1}\right)$ (since $H$ is connected). If $x_{j} \in S_{1}$, then there exists $z_{j} \in S_{1} \cap N_{G}\left(x_{j}\right)$ because $S_{1}$ is a dominating set. Since $\left\{z_{j}\right\} \times V(H) \subseteq C_{1}$, it follows that $\left\{x_{j}\right\} \times V(H) \subseteq N_{G \square H}\left(C_{1}\right)$. Hence, if $a=b$, then $\left[\left(x_{1}, a\right),\left(x_{2}, a\right) \ldots,\left(x_{k}, a\right)\right]$ is an $(x, a)-(y, b)$ path in $\left\langle N_{G \square H}\left(C_{1}\right)\right\rangle$. Suppose $a \neq b$. Let $\left[a_{1}, a_{2}, \ldots, a_{r}\right]$, where $a_{1}=a$ and $a_{r}=b$, be an $a-b$ geodesic. Then $\left[\left(x_{1}, a_{1}\right),\left(x_{2}, a_{1}\right) \ldots,\left(x_{k}, a_{1}\right),\left(x_{k}, a_{2}\right), \ldots,\left(x_{k}, a_{r}\right)\right]$ is an $(x, a)-(y, b)$ path in $\left\langle N_{G \square H}\left(C_{1}\right)\right\rangle$.

Therefore, $C_{1}$ is an nckfd-set in $G \square H$.

Similarly, if $S_{2}$ is a $k f d$-set in $H$, then $C_{2}=V(G) \times S_{2}$ is an $n c k f d$-set in $G \square H$.

Corollary 8. Let $G$ and $H$ be nontrivial connected graphs and $\varnothing \neq S_{1} \subseteq V(G)$. The following are equivalent:

(i) $S_{1}$ is a $k f d$-set of $G$.

(ii) $C_{1}=S_{1} \times V(H)$ is a kfd-set of $G \square H$.

(iii) $C_{1}=S_{1} \times V(H)$ is an nckfd-set of $G \square H$.

Proof. By Corollary 4, $(i)$ implies (ii). Suppose $C_{1}$ is a $k f d$-set of $G \square H$. Let $v \in$ $V(G) \backslash S_{1}$ and let $a \in V(H)$. Then $(v, a) \notin C_{1}$ and $\left|N_{G \square H}((v, a)) \cap C_{1}\right|=\left|N_{G}(v) \cap S_{1}\right|=k$ since $C_{1}$ is a $k f d$-set. Thus, $S_{1}$ is a $k f d$-set of $G$ and $(i i)$ implies $(i)$. By Theorem 14, (i) implies (iii). By definitions of $k f d$-set and $n c k f d$-set, $(i i i)$ implies (ii). Therefore, Statements $(i),(i i)$, and (iii) are equivalent.

Corollary 9. Let $G$ and $H$ be nontrivial connected graphs of orders $m$ and $n$, respectively, and $k$ a positive integer with $k \leq \min \{m, n\}$. Then

$$
\gamma_{n c k f d}(G \square H) \leq \min \left\{n \cdot \gamma_{k f d}(G), m \cdot \gamma_{k f d}(H)\right\} .
$$

In particular, $\gamma_{n c k f d}\left(G \square K_{n}\right) \leq \min \left\{n \cdot \gamma_{k f d}(G), m k\right\}$. 
Remark 4. The bound given in Corollary 9 is sharp. However, the strict inequality can be attained.

To see this, consider the graphs shown in Figure 6. The shaded vertices in each graph form a $\gamma_{n c k f d}$-set. Thus,

(a) $\gamma_{n c 1 f d}\left(K_{3} \square P_{2}\right)=2=\min \{2 \cdot 1,3 \cdot 1\}=\min \left\{\left|V\left(P_{2}\right)\right| \cdot \gamma_{1 f d}\left(K_{3}\right),\left|V\left(K_{3}\right)\right| \cdot \gamma_{1 f d}\left(P_{2}\right)\right\}$,

(b) $\gamma_{n c 2 f d}\left(P_{3} \square C_{4}\right)=6=\min \{4 \cdot 2,3 \cdot 2\}=\min \left\{\left|V\left(C_{4}\right)\right| \cdot \gamma_{2 f d}\left(P_{3}\right),\left|V\left(P_{3}\right)\right| \cdot \gamma_{2 f d}\left(C_{4}\right)\right\}$, and

(c) $\gamma_{n c 2 f d}\left(P_{3} \square P_{4}\right)=7<\min \{4 \cdot 2,3 \cdot 3\}=\min \left\{\left|V\left(P_{4}\right)\right| \cdot \gamma_{2 f d}\left(P_{3}\right),\left|V\left(P_{3}\right)\right| \cdot \gamma_{2 f d}\left(P_{4}\right)\right\}$.

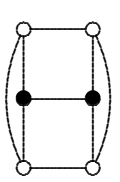

(a)

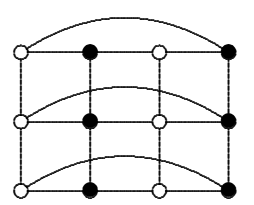

(b)

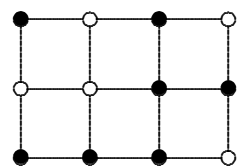

(c)

Figure 6: The graphs $K_{3} \square P_{2}, P_{3} \square C_{4}$ and $P_{3} \square P_{4}$.

\section{Acknowledgements}

This research is funded by the the Philippine Commission on Higher Education-Faculty Development Program Phase II (CHED-FDP II) and the Mindanao State University-Iligan Institute of Technology.

\section{References}

[1] S. Arumugam and C. Sivagnanam. Neighborhood Connected Domination in Graphs. Journal of Combinatorial Mathematics and Combinatorial Computing, 73:55-64, 2010.

[2] W. M. Bent-Usman D. P. Gomisong and R. T. Isla. Connected $k$-Fair domination in the Join, Corona, Lexicographic and Cartesian Products of Graphs. Applied Mathematical Sciences, 12:1341-1355, 2018.

[3] Y. Caro A. Hansberg and M. A. Henning. Fair domination in graphs. Discrete Mathematics, 19:1-18, 2011.

[4] E. Maravilla R. Isla and S. R. Canoy Jr. Fair Domination in the Join, Corona and Composition of Graphs. Applied Mathematical Sciences, 93:4609-4620, 2014. 
[5] E. Maravilla R. Isla and S. R. Canoy Jr. Fair Total Domination in the Join, Corona and Composition of Graphs. International Journal of Mathematical Analysis, 54:2677$2685,2014$.

[6] E. Maravilla R. Isla and S. R. Canoy Jr. $k$-fair Domination in the Join, Corona, Composition and Cartesian product of Graphs. Applied Mathematical Sciences, 178:88638874, 2014. 